
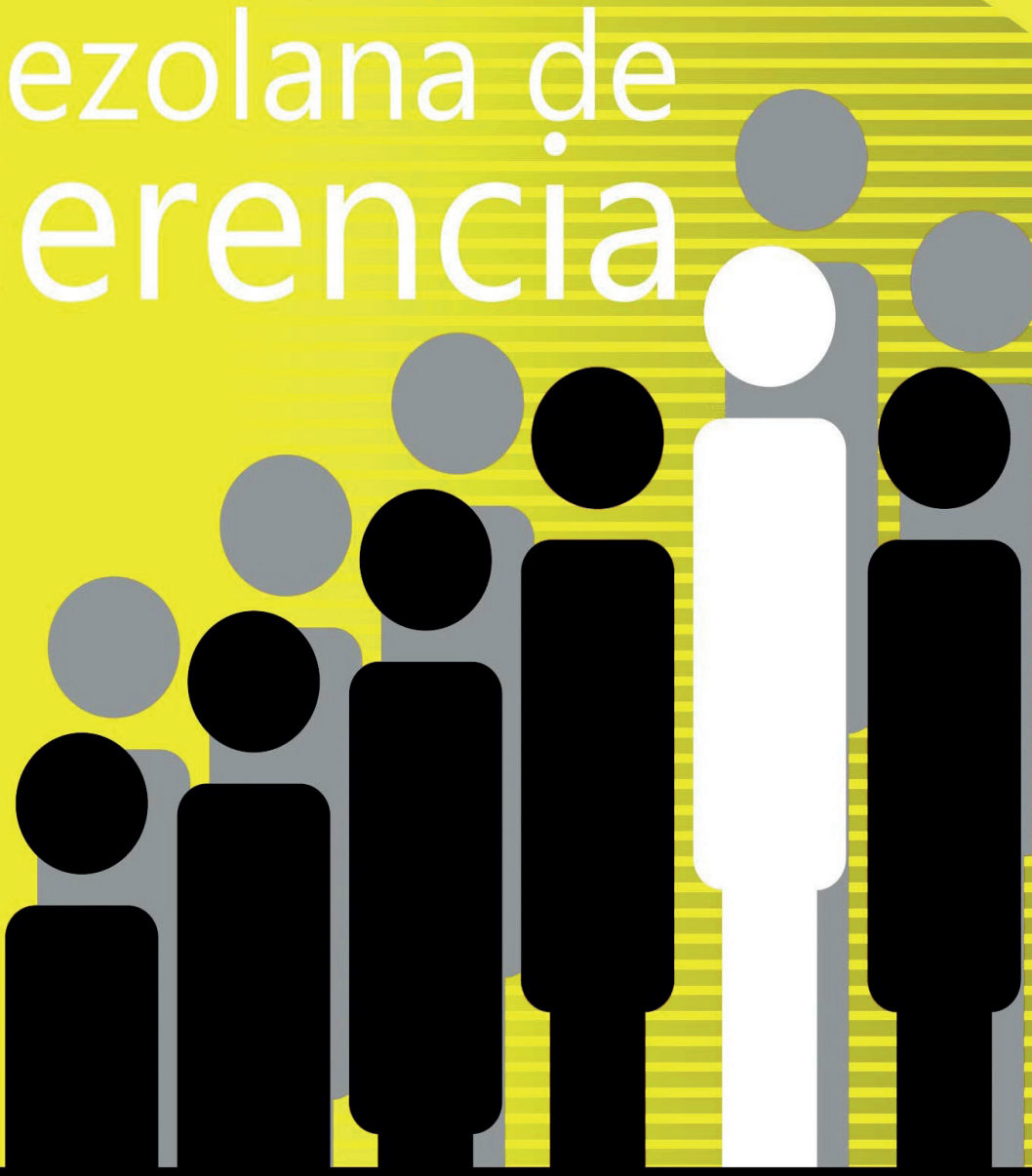


\title{
Clima organizacional y motivación laboral como insumos para planes de mejora institucional ${ }^{*}$
}

\author{
Macías García, Eva Katherine \\ Vanga Arvelo, María Giuseppina ${ }^{* *}$
}

\section{Resumen}

En las instituciones, no solo se generan lazos laborales sino también del tipo social y afectivo que, aunado a las jornadas laborales extensas, hacen que de manera imperiosa se requiera de un ambiente laboral propicio, seguro y confortable. El objetivo de esta investigación es el de hacer un diagnóstico situacional basado en los docentes de la carrera de Ingeniería Civil de la Universidad Técnica de Manabí, mediante una investigación de campo, con enfoque cuantitativo y muestreo censal, para la aplicación de un cuestionario cuyos resultados fueron analizados bajo la técnica de semaforización; se determinaron dimensiones como clima organizacional referido a lo externo del trabajador y la motivación laboral referido a lo interno. Los resultados muestran que hay deficiencias asociadas al clima organizacional, pero que estas no influyen en la motivación de los trabajadores. Se concluye que los diagnósticos realizados a las organizaciones deben servir de insumos para la elaboración de planes de mejora que se centren en los aspectos desfavorables encontrados. Se recomienda que estos planes se constituyan en acciones inmediatas, a corto, mediano, y largo plazo según los resultados y que se mantengan incluso aquellos aspectos encontrados en estado óptimo.

Palabras clave: clima organizacional; desempeño laboral; motivación laboral; Carrera de Ingeniería Civil de la Universidad Técnica de Manabí.

* Este artículo es el resultado de una investigación llevada a cabo en la Universidad Técnica de Manabí a los docentes de la carrera de Ingeniería Civil realizada en el año 2020 sin financiamiento.

** Eva Macías: Economista y Secretaria de la Carrera de Ingeniería Química de la Universidad Técnica de Manabí. E-mail: eva.macias@utm.edu.ec ORCID: https://orcid.org/0000-0003-2739-8648

*** María Vanga: PhD en Arquitectura y docente de Arquitectura de la Universidad Técnica de Manabí. E-mail: maria.vanga@utm.edu.ec (Correspondencia) ORCID: https://orcid.org/0000-0003-0143-8381 


\title{
The organizational climate and labor motivation as inputs for institutional improvement plans
}

\begin{abstract}
In institutions, not only labor ties are generated but also social and affective, together with long working hours, urgently require an auspicious, safe and comfortable work environment. The objective of this research is to make a situational diagnosis based on the professors of Civil Engineering career in the Universidad Técnica de Manabí, through a field research, with a qualitative approach and census sampling, for applicating a questionnaire whose results were analyzed using the traffic light technique; dimensions were determined such as organizational climate referred to the worker's external and work motivation referred to the worker's internal. The results show there are deficiencies associated with the organizational climate, but these do not influence the motivation of workers. It is concluded the diagnoses made to the organizations should serve as inputs for the elaboration of improvement plans that focus on the unfavorable aspects found. It is recommended these plans be constituted in immediate, short, medium, and longterm actions according to the results and even those aspects found in optimal condition should be maintained.
\end{abstract}

Key Words: organizational climate; job performance; motivation for work; Universidad Técnica de Manabí's Civil Engineering Degree.

\section{Introducción}

A pesar de que las universidades públicas son consideradas organizaciones democráticas, su desenvolvimiento es complejo, por lo que la convivencia a lo interno también lo es. Los docentes pasan la mayor parte del tiempo inmerso en el trabajo, las clases, reuniones y otras actividades, que los hacen enfocarse en cumplir con lo meramente solicitado y a no estar motivados en ser proactivos.

En este sentido, estudiar como es el clima organizacional y como está la motivación al interno universitario es de gran importancia para así poder identificar las necesidades, saber si se está encaminado en los objetivos organizacionales y poder trazar estrategias y acciones que permitan ejecutar la misión y visión institucionales.

El clima organizacional para Alfaro, "se refiere al ambiente en el cual se relacionan los miembros de la empresa o institución y asimismo a como está constituida y estructurada la misma, esto se refiere al modo y la forma de trabajo" (2019: 6). Según Borja, el "clima organizacional es conexo con la motivación de los colaboradores de cada organización, y mientras la motivación de estos sea alta, el clima proporciona satisfacción, ánimo, interés 
y colaboración" (2021: 17).

La motivación laboral para Atoche, es "la fuerza o impulso interno que nos mueve a realizar una determinada tarea, para lo cual ocupamos de manera voluntaria nuestros recursos físicos y mentales hasta lograr la meta deseada" (2019: 16). Sin embargo, para Peña y Villón es visto desde una perspectiva externa indicando que es "el resultado de la interrelación del individuo y el estímulo realizado por la organización con la finalidad de crear elementos que impulsen e incentiven al empleado a lograr un objetivo" (2018: 185). Para efectos de esta investigación se tomará a la motivación laboral como factor interno y al clima organizacional como factor externo.

Se han hecho varios estudios al respecto, uno en España referente al clima organizacional y satisfacción laboral, concluyendo que "el clima es un constructo colectivo" (Pecino-Medina et al, 2015: 663). Lo que es percibido del clima tiene una gran importancia ya que permite el enlace entre las prácticas, políticas y procesos de gestión. El clima se conecta con los estados cognitivos y afectivos en relación positiva con la satisfacción laboral, su importancia está centrada en el efecto que produce sobre la conducta, considerada esta como muy importante para la eficiencia organizacional y el desempeño (ibid).

En Perú, investigaciones han revelado que la percepción que tienen los docentes sobre el clima organizacional es favorable en tanto existan buenas relaciones interpersonales entre ellos y mejor aún si predomina la buena comunicación y el optimismo. Se detectó que la remuneración percibida no estaba acorde con la sobrecarga laboral, que existe ausencia de incentivos y que se debe mejorar la relación entre docentes y directivos. Recomiendan, "continuar la lucha por la mejora de la educación, el incremento de las remuneraciones, la promoción de incentivos, el optimismo, la buena comunicación y la constante capacitación... a través de planes y políticas concretas y viables" (Torres \& Espino, 2020: 20).

Un estudio realizado en Ecuador, indica que el clima organizacional no influye en el desempeño docente, sin embargo, es de gran importancia ya que, por medio de la creación de un clima positivo, el nivel de desempeño puede ser elevado alcanzando los objetivos planteados (Lainez, 2019).

La carrera de Ingeniería Civil de la Universidad Técnica de Manabí (UTM), no cuenta al momento con un estudio de este tipo, es por ello que el objetivo de esta investigación es hacer un diagnóstico que refleje el clima organizacional y el grado de motivación de sus docentes, con la finalidad de emitir recomendaciones para los planes de mejora institucional. Si se tiene una retroalimentación de procesos y su afectación con el comportamiento, se tendrán insumos para que los planes estén orientados a cambios de actitudes y conductas, se detectarán debilidades y se podrá así, a través del diagnóstico, fusionar ambos resultados permitiendo el cierre de brechas institucionales.

\section{Aportes metodológicos de la investigación}

Eltipo de investigaciónes decampo, ya que la recopilación de información es obtenida de los sujetos directamente (datos primarios), sin ejercer control ni alteración (Chimbosina, 2020). El enfoque utilizado es el cuantitativo, que, según Hernández, Fernández y Baptista (2010), es característico de acciones 
con determinada secuencia sometidas a prueba; las ideas originarias con sus parámetros establecidos derivan en preguntas y objetivos que orientan el estudio.

Debido a que la población no es extensa, se utilizó un muestreo censal, es decir, la población y la muestra son la misma (García, 2019), conformada por 33 individuos (incluidos el Vicedecano, Coordinador y 31 docentes).

La recolección de datos se hizo en el segundo semestre del 2020. Se operacionalizaron diversos conceptos o variables, siendo estas todo aquello que va a estar sujeto a estudio, medición y control; es un concepto clasificatorio al poder asumir valores diferentes y a la vez poder ser definidas conceptual y operacionalmente (Nuñez, 2007). Esto permitirá construir instrumentos a la medida, convirtiendo indicadores en ítems, mostrando en forma esquemática el contenido de la investigación y permitiendo tener una visión global (Reguant \& Martínez-Olmo, 2014).

Las dimensiones que organizan la información son: general, clima organizacional y motivación laboral. Para la dimensión general se tomó en cuenta la edad, sexo, grado de instrucción, categoría y tiempo de servicio. Para la dimensión clima organizacional se consideraron varios aspectos como la motivación (manifestada en aspectos tangibles como ascenso, salario, entre otros), las condiciones del espacio, el clima entre compañeros, estilos de dirección, motivación de las autoridades, carga académica, compañerismo, evaluación del desempeño, relación con las autoridades, la capacitación, materiales y recursos, criterios de evaluación, expectativas sobre el empleado (considerados externos al individuo). Para la dimensión motivación laboral, la medición se hizo en base al compromiso, identificación del trabajador y su entusiasmo (indicativos internos del estado del trabajador).

Como medio de acercamiento a la realidad estudiada y reducirla a una representación, facilitando de esta manera su análisis y haciéndola más comprensible (Fassio \& Pascual, 2016), se usó el cuestionario como "técnica de recogida de información sobre opiniones, actitudes, habilidades, aplicables a amplias muestras representativas de poblaciones definidas" (Martínez, 2014: 244).

Está conformado por preguntas cerradas (definidas previamente las alternativas de respuestas de selección y dicotómicas); preguntas con escalas de satisfacción y acuerdo (que reflejan la valoración del encuestado y nivel de satisfacción), midiendo actitudes con grados de acuerdo y desacuerdo (Múria \& Gil, 1998). Tiene abiertas sin estructura, donde se responde libremente (Malhotra, 2004).

Para las respuestas de satisfacción y acuerdo y dicotómicas, se utilizará un sistema de semaforización, técnica utilizada para analizar los resultados y catalogarlos en niveles (Jiménez \& Mosquera, 2017). Los niveles se muestran en la tabla 1. 


\section{Tabla 1}

\section{Parámetros de medición para la semaforización}

\begin{tabular}{ccl}
\hline Intervalo & Nivel & Estado - Acciones a aplicar \\
\hline $0-20 \%$ & Critico & Deplorable - Urgentes \\
\hline $21-40 \%$ & Riesgo & Mal estado - Corto plazo \\
\hline $41-60 \%$ & Preventivo & Comienzo del deterioro - Mediano plazo \\
\hline $61-80 \%$ & Adecuado & Buenas condiciones - Largo plazo \\
\hline $81-100 \%$ & Óptimo & Excelente - Mantenimiento \\
\hline
\end{tabular}

Fuente: elaborado a partir de los intervalos propuestos por Pérez, 2014: 97.

Los valores a ser tomados en cuenta como puntos de partida de las respuestas son los estados deseables. Por ejemplo, en la escala de agrado: "Muy agradable", "Agradable", "Poco agradable" y "No agradable", lo ideal es que las respuestas se perfilen hacia el "Muy agradable", por lo que el porcentaje de frecuencia a tomar para valorar el estado de si es crítico, riesgo, preventivo, adecuado u óptimo, será "Muy agradable"; así para las demás escalas y preguntas dicotómicas.

Para las de selección, se tomará como respuesta aquellas que tengan mayor incidencia o frecuencia. Para las abiertas se codificará sistematizando las respuestas y categorizándolas; estas preguntas ofrecen detalle y profundidad a nivel personal (Feixas, 2006). En la tabla 2 se presenta un resumen de la forma de valoración de los diferentes tipos de preguntas encontradas en el cuestionario aplicado.

\section{Tabla 2}

\section{Métodos de valoración para analizar el cuestionario}

\begin{tabular}{lll}
\hline Tipo de pregunta & Opciones a considerar & Valoración \\
\hline $\begin{array}{l}\text { Escala de satisfacción } \\
\text { y de acuerdo }\end{array}$ & $\begin{array}{l}\text { Muy agradable, Muy aceptable, Muy competitivo, Muy cordial, } \\
\text { Totalmente de acuerdo }\end{array}$ & Semaforización \\
\hline Dicotómicas & $\mathrm{Si} / \mathrm{No}$ & Semaforización \\
\hline Selección & Ejemplo: Temperatura, lluminación, Ruido, Ergonomía & $\begin{array}{l}\text { Mayor inciden- } \\
\text { cia }\end{array}$ \\
\hline Abiertas & Contenido diverso en forma de relatoría o de lista & Categorización \\
\hline
\end{tabular}

Fuente: Elaboración propia. 


\section{Clima organizacional y Motivación laboral: Aproximaciones teóricas}

Para hacer el abordaje teórico de las dos dimensiones principales usadas en el diagnóstico organizacional se recurrió a varios autores, estableciéndose los indicadores señalados en el apartado anterior.

\subsection{Clima organizacional}

Para Chávez, Escudero y Delfín (2016), hay tres definiciones de importancia como las dadas por Forehand y Gilmer en el año 1964 donde definen al clima organizacional como las características que perciben los trabajadores y que hacen distinguirlas de otras organizaciones; la de Taguiri y Litwin en 1968, como el conjunto de interpretaciones de los empleados sobre su organización, y que impactan en su motivación y actitud; y la de Campbell, Dunnette, Lawler y Weick en el 1970, expresada en el conjunto de atributos que pueden ser inducidos en mutuo acuerdo entre la organización y sus miembros.

Ramos (2012), indica que el instrumento que se usa con más frecuencia para evaluar el clima organizacional es el cuestionario de Likert, cuyos elementos son los métodos de mando, las fuerzas motivacionales, diferentes procesos (comunicación, influencia, de tomas de decisiones, de planificación, de control), y los objetivos de rendimiento y perfeccionamiento. Menciona al cuestionario de Litwin y Stringer que miden la estructura, responsabilidad individual, remuneración, riesgos y toma de decisiones, el apoyo, y tolerancia al conflicto. También refieren el cuestionario de Schnedider y Bartlett que mide la estructura, la implicación con nuevos empleados, los conflictos interagencias, la autonomía de los empleados, y el grado de satisfacción general. Pritchard y Karasick plantearon la autonomía, la estructura, la remuneración, el rendimiento, la motivación, el estatus, la flexibilidad e innovación, centralización de la toma de decisiones, y el apoyo.

\subsection{Motivación laboral}

Para Castillo (2021), hay varias definiciones de gran importancia sobre la motivación laboral como la dada por Benavides en el 2017, quien considera que es un aspecto importante en los contextos laborales, ya que ayuda a determinar aquellas actividades que los empleados realizan con mayor perseverancia incidiendo en la eficiencia y los resultados de la organización.

Para Castillo (2018), la motivación se mide a través de sus características como la intensidad (cantidad de esfuerzos, resultados y desempeño), la dirección (beneficios, calidad del esfuerzo y cumplimiento de metas), y la persistencia en el trabajo (persistencia de tiempo, cumplimiento de objetivo y ejecución de metas). Estos tópicos contrastan con lo indicado por Peña (2015), quien indica que la gestión de la motivación gira en torno a el compromiso, que una vez logrado se obtienen resultados óptimos, que el futuro de la motivación laboral está enfocado en estrategias orientadas al compromiso; solo cuando el compromiso es palpable es que se pueden obtener altos niveles de desempeño. Por su lado, Delgado (2020), indica que cuando hay un alto nivel de identidad corporativa se considera como un estímulo para incrementar la motivación 
de los miembros de una organización; esta identidad personifica la ética $y$ las actitudes de la empresa y de los empleados y quienes la comparten están unidos por ese vínculo. Para Flores et al, el entusiasmo es un constructo motivacional que integra sentimientos de vigor, absorción y dedicación.

\section{Clima organizacional y Motivación laboral en la carrera de Ingeniería Civil de la Universidad Técnica de Manabí}

La UTM es una universidad pública ubicada en la Provincia de Manabí, ciudad Portoviejo, en Ecuador. Fue fundada en octubre del año 1952 por el Dr. José María Velasco Ibarra. Interviene en los sectores público y privado, y actualmente está acreditada por el Consejo de Aseguramiento de la Calidad de la Educación Superior (CACES). Está conformada por 10 facultades, el Instituto de Ciencias Básicas y el Instituto de Postgrado.

La carrera de Ingeniería Civil fue creada en mayo de 1970 para dar respuesta al desarrollo de obras hídricas de Manabí y del país; está ubicada en la Facultad de Ciencias Matemáticas, Físicas y Químicas, creada en octubre del año 1958.

El diagnóstico situacional aplicado a la carrera de Ingeniería Civil arrojó los resultados que se muestran a continuación.

\subsection{Dimensión general}

Esta dimensión refleja que las edades comprendidas entre los 41 y 50 años es la más recurrente con un total de $37.5 \%$, donde el $84.4 \%$ son hombres, la mayoría tienen grado de instrucción de Magister $(71.9 \%$ ), el $43.8 \%$ tiene categoría de Titular Principal y el $46.9 \%$ tiene más de 10 años trabajando en la institución.

Lo más importante de estos resultados es que las mujeres tienen porcentajes menores de participación. Según el Boletín Anual de agosto 2020 de la Secretaría de Educación Superior, Ciencia, Tecnología e Innovación (SENESCYT), para el año 2018 el $60 \%$ de los docentes eran hombres (universidades y escuelas politécnicas); mientras que el $40 \%$ mujeres, destacando que desde el año 2015 este porcentaje se incrementó en solo dos puntos porcentuales (Rosales, 2020).

Esto sucede también a nivel de estudiantes; en el 2017, la escuela Politécnica Nacional hizo una encuesta a sus graduados de Ingeniería Civil y resulto el $75 \%$ ser de género masculino (Angulo, 2017). Así mismo, en la Universidad Central del Ecuador, indican que la Ingeniería Civil es una carrera predominantemente masculina $(73.43 \%)$, reflejando que las mujeres escogen carreras científicas en una escala menor a los hombres (Ramírez, Manosalvas \& Cárdenas, 2019).

Se sugiere reformar la presencia femenina dentro del ámbito de las ingenierías, dando a conocer modelos ejemplo que animen a las mujeres a escoger las carreras de su preferencia sin importar que estén conformadas mayormente por hombres, así como a dedicarse a la docencia en las mismas; y a través del contacto con las empresas y la vinculación, promover la inserción laboral de las mujeres en estos campos.

\subsection{Clima organizacional}

En la dimensión clima 
organizacional (CO), relacionada con el estímulo que recibe el individuo desde el exterior, fueron valorados varios aspectos (ver tabla 3).

\section{Tabla 3}

\section{Preguntas procesadas por mayor incidencia y categorización -} dimensión $\mathrm{CO}$

\begin{tabular}{|c|c|c|}
\hline Factor & Ítem & Frecuencia \\
\hline \multirow{6}{*}{ Motivación } & $\begin{array}{l}\text { Ascenso / Promoción, Mejora de salario, Crecimiento } \\
\text { profesional, Reconocimiento }\end{array}$ & $12.5 \%$ \\
\hline & $\begin{array}{l}\text { Ascenso / Promoción, Crecimiento profesional, Buen ambiente, } \\
\text { Plan de formación }\end{array}$ & $9.4 \%$ \\
\hline & $\begin{array}{l}\text { Ascenso / Promoción, Crecimiento profesional, } \\
\text { Reconocimiento, Plan de formación }\end{array}$ & $6.3 \%$ \\
\hline & $\begin{array}{l}\text { Ascenso / Promoción, Mejora de salario, Crecimiento } \\
\text { profesional, Plan de formación }\end{array}$ & $6.3 \%$ \\
\hline & $\begin{array}{l}\text { Crecimiento profesional, Buen ambiente, Plan de formación, } \\
\text { Comunicación }\end{array}$ & $6.3 \%$ \\
\hline & $\begin{array}{l}\text { Mejora de salario, Crecimiento profesional, Reconocimiento, } \\
\text { Buen trato }\end{array}$ & $6.3 \%$ \\
\hline \multirow{5}{*}{$\begin{array}{l}\text { Mejora a } \\
\text { espacio }\end{array}$} & Temperatura, Ruido, Mobiliario & $18,8 \%$ \\
\hline & Temperatura, Iluminación, Mobiliario & $17,2 \%$ \\
\hline & Temperatura, Mobiliario & $17,2 \%$ \\
\hline & Temperatura & $15,6 \%$ \\
\hline & Temperatura, Ruido & $15,6 \%$ \\
\hline
\end{tabular}

Fuente: Elaboración propia.

Se plantearon nueve motivaciones que resulten esenciales para el desempeño laboral para seleccionar cuatro, de las cuáles, las que más recurrencia tuvieron fueron el ascenso, la mejora salarial, el crecimiento profesional y el reconocimiento.

En cuanto al ascenso y/o promoción, Solórzano (2020) indica que el incentivo trasciende el dinero asociándose a veces con un posible ascenso. Esta falta de ascenso puede generar desmotivación relacionada con el fracaso al permanecer en el mismo puesto de trabajo (Espinoza, 2020). Por otro lado, los bajos salarios están catalogados como una de las principales causas de insatisfacción. Hay factores que no son controlados por el docente como las condiciones laborales, interponiéndose esto en el correcto desempeño de sus funciones, aunado a la carga excesiva de trabajo y el mencionado bajo salario (Arreiza, Restrepo \& Osorio, 2020).

En cuanto al reconocimiento, su 
falta desmotiva y afecta negativamente el desempeño señalan Álvarez y Ayala (2020); indican que según la teoría de Maslow y su pirámide de jerarquía de necesidades, el cuarto nivel (de abajo hacia arriba comenzando por las necesidades fisiológicas), corresponde a la necesidad de reconocimiento, y esto tiene una influencia profunda sobre el comportamiento. Un empleado al ganar reconocimiento piensa que su labor es productiva haciendo fácil el establecimiento de metas.

Llama la atención que el factor presente en todas las motivaciones que tienen mayor frecuencia es el crecimiento profesional. Para Palma y Hermosillo (2020), este crecimiento es uno de los componentes de la satisfacción laboral. Tapia (2020), afirma que hay una tendencia a abandonar los puestos laborales cuando no se cuenta con un adecuado plan de carrera o crecimiento profesional.

En cuanto a los espacios, se plantearon cinco alternativas para seleccionar un máximo de tres. El orden de incidencia de mayor a menor fue temperatura, mobiliario, ruido e iluminación, resaltando la temperatura, presente en todas las elecciones.

Para los docentes de la carrera de Ingeniería Civil, lo prioritario a resolver es la temperatura. La universidad, se encuentra ubicada en la Provincia de Manabí, Portoviejo; con un promedio de temperaturas altas, registrándose en el año $201834^{\circ} \mathrm{C}$ con sensación térmica de $40^{\circ} \mathrm{C}$ (Mina, 2018). La humedad es otro factor importante y en Portoviejo, el período más húmedo dura 9.8 meses, alcanzándose 100\% (Weather, 2021). Es necesario hacer un estudio de confort ya que Riquelme (2021) afirma que "la temperatura ideal en la oficina se encuentre entre los 20 y los 24 grados.
Igualmente, se aconseja un nivel de humedad entre el $20 \%$ y el $60 \%$ ".

El mobiliario es otro tema de importancia, asociado con el confort y desempeño eficiente además de incidir en la salud del trabajador. Las jornadas laborales ocupan aproximadamente un $33,33 \%$ del total del día, siendo un porcentaje significativo. Aumentar el rendimiento en el trabajo se supedita al mobiliario de la oficina; además de que un mobiliario ergonómico aumenta la productividad en un 10\% (Gedesa, 2019).

Por otro lado, el confort acústico también es un aspecto relevante, ya que el ruido impide al trabajador concentrarse al no tener capacidad de abstracción. Para Ruiz (2020), el denominado ruido ocupacional está presente en todos los trabajos y puede llegar a ocasionar efectos contraproducentes no auditivos como psíquicos y sociales, aunque en oportunidades no ocasione riesgos.

La iluminación en el caso de estudio, hace referencia a la incidencia del sol al entrar por las ventanas de las oficinas. Debe existir un equilibrio de la luz natural ya que esta se refleja en las pantallas de las computadoras y produce deslumbramiento o reflejos inadecuados que pueden ser nocivos. Yamin et al, (2020), indican que, si el control solar es inadecuado, se tiende a bloquear la iluminación natural evitando el deslumbramiento y usando luz artificial, aumentando el consumo de energía; en los climas donde los cielos son claros, hay una gran disponibilidad de luz natural pudiendo ser aprovechada para el ahorro energético, sin embargo, es necesario un adecuado control solar lumínico y una adecuada correlación entre ellos.

Se recomienda hacer un estudio de las condiciones de las oficinas, la 
incidencia de la luz natural y artificial, temperatura, humedad, contaminación acústica y ventilación cruzada. Las oficinas de la institución son amplias, sin embargo, debería revisarse la distribución interna del mobiliario y que éste sea ergonómico, adaptado a las necesidades particulares de cada docente. Un aspecto que debe ser corregido es la atención a alumnos; las filas de alumnos a la espera de atención en las afueras de las oficinas, irrumpe con la tranquilidad y concentración de otros docentes, recomendándose destinar lugares especiales para dicha tarea. Es necesario hacer autogestión para la colocación de aires acondicionados en las oficinas, aulas y laboratorios, además de cortinas que eviten el deslumbramiento.

Se sugiere implementar modelos de compensación laboral, que ayuden en la retención de personal, además de tomar en cuenta la retribución indirecta. En países como Ecuador, donde las universidades cuentan con bajos presupuestos, el aumento de las remuneraciones sería dificultoso, sin embargo, los ascensos y escalafón podrían promoverse. Para las retribuciones indirectas se puede promover la asignación de días libres; capacitaciones elegidas por el docente; entrega de diplomas por desempeño; reconocimientos por proyectos, artículos o libros; asignación de horas docentes acorde a su producción científica; flexibilidad en horario; promoción, entre otros. Otros aspectos estudiados en la dimensión clima organizacional pueden visualizarse en la tabla 4.

\section{Tabla 4}

Preguntas procesadas por semaforización - dimensión CO

\begin{tabular}{|c|c|c|}
\hline Factor & Valoración & Lectura \\
\hline Clima entre compañeros (Muy competitivo) & $6,3 \%$ & \multirow{2}{*}{ Crítico } \\
\hline Estilo de dirección (Muy aceptable) & $15,6 \%$ & \\
\hline $\begin{array}{l}\text { Existen motivaciones suficientes por parte de las autoridades para } \\
\text { mejorar el desempeño laboral (Totalmente de acuerdo) }\end{array}$ & $28,1 \%$ & \multirow{4}{*}{ Riesgo } \\
\hline Espacio físico (Muy agradable) & $29,7 \%$ & \\
\hline La carga académica repercute en la calidad y productividad (No) & $35,95 \%$ & \\
\hline Compañerismo (Muy agradable) & $37,5 \%$ & \\
\hline $\begin{array}{l}\text { Contribución de la evaluación de desempeño en la mejora de debili- } \\
\text { dades (Totalmente de acuerdo) }\end{array}$ & $43,8 \%$ & \multirow{4}{*}{ Preventivo } \\
\hline Relación con las autoridades (Muy cordial) & $43,8 \%$ & \\
\hline Capacitación permite innovar (Totalmente de acuerdo) & $53,1 \%$ & \\
\hline Cuenta con materiales y recursos (Si) & $59,4 \%$ & \\
\hline Se conocen los criterios de evaluación de desempeño (Si) & $75 \%$ & \multirow{2}{*}{ Adecuado } \\
\hline Estar de acuerdo con la forma de ser evaluado (Si) & $76,55 \%$ & \\
\hline Conoce lo que esperan las autoridades del empleado (Si) & $96,9 \%$ & Óptimo \\
\hline
\end{tabular}

Fuente: Elaboración propia. 

institucional

Uno de los estados que más resalta al estar en la categoría de deplorable es el clima entre compañeros con estado crítico y que amerita acciones urgentes. No sentir que hay competitividad puede ser nocivo al haber la tendencia de no estar motivado para superarse. Según Manzano (2020), todo profesional vive en un ambiente competitivo y es precisamente éste el que hace que el individuo se vea obligado y motivado a prepararse continuamente, encarando nuevas tecnologías y retos académicos para así poder hacer un aporte en el crecimiento de la sociedad.

Por otro lado, el estilo de dirección es una variable proporcional al buen desempeño de organizaciones; este estilo es considerado como la interacción que existe entre subordinados $y$ dirigentes $y$, por consiguiente, influye de manera significativa en sus procesos. Para Castiblanco et al, el estilo de dirección es "un elemento clave para alcanzar el pleno y satisfactorio rendimiento del colaborador; la baja valoración de esta dimensión da muestra de los constantes cambios de estado de ánimo del personal" (2020: 25).

Seguidamente, encontramos los factores que están poniendo a la organización en estado de riesgo y que requieren acciones a corto plazo como el que existan motivaciones suficientes por parte de las autoridades para mejorar el desempeño laboral de los trabajadores. Anteriormente se indicó, que las motivaciones como ascenso, mejora salarial, crecimiento profesional y reconocimiento, eran consideradas por los encuestados como esenciales para su desempeño laboral, pudiendo deducir que estos aspectos no han sido cubiertos. Los trabajadores que tengan suficiente motivación serán eficientes y tendrán éxito en el desempeño de sus labores, darán lo mejor de sí y se sentirán satisfechos. Según Villalba (2020), la motivación es una retroalimentación donde el empeño del trabajador se ve energizado y encausado hacia el logro de metas; la calidad del clima organizacional influye en las emociones de los empleados, siendo fundamental que sea agradable y positivo.

En cuanto a los espacios, Pichón y Vega (2020), indican que los empleadores son responsables en ofrecer un espacio confortable. Estos espacios abarcan los de docencia, gestión, investigación, vinculación y actividades administrativas; para Maldonado (2020), los espacios físicos adecuados posibilitan la ejecución de actividades laborales de manera eficiente y eficaz.

En cuanto a la carga académica, las acciones a tomar a corto plazo incidirán en el desempeño docente. Los resultados mostraron que los encuestados sienten que la carga de horas docentes no es la adecuada y eso repercute en la calidad de su trabajo y productividad; tal y como expresa Cetzal et al, (2020), está considerada como un factor que afecta el desempeño docente, incidiendo en su participación y compromiso con los objetivos institucionales y también en los proyectos.

Del mismo modo, está el compañerismo, en donde estudios demuestran que la satisfacción laboral influye de manera positiva en el desempeño laboral, siendo uno de los indicadores más importantes el compañerismo. Indican que las personas del trabajo obtienen mucho más que logros o remuneración, incluyendo el cubrir la necesidad de interactuar socialmente; la relación laboral es relevante para la motivación (Chelton \& Balladares, 2020). Compañerismo 
implica cohesión, armonía, respeto y apoyo, y para lograrlo es necesario una buena comunicación, fomentar el conocerse y propiciar un ambiente de confianza. Las críticas son importantes para el crecimiento personal y profesional y deben ser constructivas y darse de manera personal, en forma conciliadora y constructiva.

En estado preventivo y que amerita acciones a mediano plazo tenemos cuatro factores, como la contribución de la evaluación de desempeño en la mejora de habilidades; uno de sus fines tangibles es detectar debilidades y tomar acciones para convertirlas en fortalezas. Según Moreno (2019), este proceso de evaluar el desempeño consiste en valorar cualitativa y cuantitativamente los aportes que los empleados hacen a la organización, así como también el grado de eficiencia con el que llevan a cabo sus tareas y funciones. Cuando se valora la ejecución de funciones y su eficacia, se desprenden los puntos y áreas que deben de ser tomados en cuenta para las mejoras; de esas áreas se suscitan los ítems a ser incluidos en capacitaciones. $\mathrm{Y}$ es que, a propósito de las capacitaciones, este es uno de los rubros mencionados en estado preventivo: una capacitación que permita la innovación.

Según Berlanga et al, la capacitación debe de ir más allá de la mera formación, representando un reto para el sistema educativo la formación de personas responsables y capaces de solventar los problemas del contexto, "pero esto solo puede cumplirse con docentes capacitados profesional y pedagógicamente capaces de convertirse en verdaderos agentes de cambio que motiven el desarrollo de competencias en el estudiante" (2020: 103).
Para Rojas (2018), una de las actividades de mayor importancia en la cultura organizacional es la capacitación, además de ser una práctica que forja el camino hacia el éxito institucional. La capacitación mejora el desempeño laboral y otros aspectos, teniendo la evaluación del desempeño la obligación de dar a conocer las debilidades de los sectores y de la estructura en donde la formación debe ser reforzada; la capacitación tiene una influencia poderosa en el desarrollo profesional, motivación ésta más seleccionada entre los encuestados. Sin embargo, se considera que la capacitación tiene que venir acompañada de los materiales y recursos adecuados para el buen desempeño docente, tercera premisa que refleja requerir atención a mediano plazo en este estudio.

Los materiales y recursos requieren inversión por parte de las instituciones. Tal y como lo indica Valdez (2013), la mayor parte del desarrollo de la tecnología y ciencia se lleva a cabo en instituciones de educación superior, lo que lleva a los países a ser más competitivos si invierten en ellas. Los problemas que enfrentan estas instituciones son semejantes, encontrándose presupuestos ineficientes y austeros, y la inequidad por parte de los gobiernos en la asignación de recursos.

$\mathrm{Y}$, por último, está la relación con las autoridades, que denota el comienzo de deterioro de la unidad en estudio y que requiere acciones a mediano plazo. Las relaciones interpersonales en una organización son uno de los aspectos más importantes ya que permiten el buen entendimiento, mayor fluidez en la comunicación, mayor empatía y confianza, crean ambientes satisfactorios y un clima agradable y armonioso. Por su parte, Cevallos y Rodríguez indican 
que "la falta de habilidades sociales y la ausencia o pobreza en las relaciones interpersonales genera sentimientos de incompetencia, aislamiento social y malestar psicológico" (2018: 24).

Siguiendo con los resultados se tiene que, factores concernientes a la evaluación también deben ser tomados en cuenta a largo plazo, como el hecho de conocer los criterios de evaluación y estar de acuerdo con la forma en ser evaluados. $Y$ es que, además de mostrar las debilidades como insumo para capacitaciones, según Albarracín y Gómez, se espera de la evaluación de desempeño que garantice mejores resultados no solo en indicadores fundamentales sino además en el desempeño humano; indican en relación a la evaluación de desempeño de los docentes que "hay una tendencia a encontrar fuertes cuestionamientos dado que los mismos cuentan con un deficiente nivel de integración a los procesos de la gestión de la institución educativa, el desarrollo profesional y la docencia cualificada" (2021: 7).

La evaluación debe sentar sus bases en lo que se deriva de ella para permitir hacer mejoras profundas a nivel académico y personal, incidiendo positivamente en el desempeño de la institución. Un punto a favor encontrado es el hecho de que los docentes saben lo que las autoridades esperan de ellos, por lo que las estrategias usadas hasta entonces de comunicación, relacionadas a las expectativas de las autoridades en relación a las actividades de docencia, gestión e investigación, son efectivas y deben mantenerse en el tiempo, incluso ser reforzadas.

Se recomienda fomentar el compañerismo a través de la promoción del trabajo en equipo, trabajo colaborativo, escucha activa, promover encuentros fuera del ámbito laboral, encausar a la unidad en un mismo bien común, evitar rivalidades, entre otros. Además, crear una competitividad sana que impulse el desarrollo y crecimiento de los docentes, por lo que las actividades mencionadas, permitirán que las posibles fricciones sean subsanadas. Para impulsar la competitividad, debe reconocerse la diversidad y dar paso a que los trabajadores exploren sus potenciales, evitar la competencia innecesaria, establecer límites sanos, evitar comparaciones, premiar logros, fijar roles y metas claras y concretas, entre otros.

Aprovechar las evaluaciones de desempeño en mejorar las debilidades encontradas es otra sugerencia, generando una capacitación que permita innovar y centrada en las preferencias del docente. Si de la evaluación no se desprende un plan de capacitación y un plan de mejoras, esta sería una actividad que carecería de sentido. La capacitación deberá ser continua, basado en necesidades reales, orientada al logro de objetivos institucionales, además de promover la calidad en sus resultados. Para desarrollar planes de carrera, se recomienda que el docente establezca sus propios objetivos, así como los que las autoridades requieren de él. Se sugiere el trazado de recorrido y estadía por varias áreas, lo que permitirá enriquecer sus habilidades, además de experimentar a cuál área se ajusta más.

En cuanto a las autoridades, se recomienda dejar atrás los liderazgos autocráticos y burocráticos, e incursionar en estilos combinados como liderazgo participativo y transformacional, considerado este último como uno de los más recomendados ya que el líder, "genera cambios en la conciencia y en el comportamiento de sus seguidores, 
orientándolos a realizarse a sí mismo, a ver las oportunidades y a resolver los desafíos del entorno, dando nuevas soluciones a los problemas que se presentan" (Zegarra, 2021: 16).

Otro aspecto a sugerir son las relaciones cordiales con todas las autoridades, no solo con los jefes inmediatos. Las autoridades deben ser vistas como pares que han alcanzado otras metas, y que por su liderazgo y trayectoria están en los cargos que ocupan. Esta visión de par, aunado con una buena y sana comunicación, hará que los trabajadores se sientan incluidos.

Todos estos aspectos mencionados para ser introducidos en los planes de mejora y ser analizados y concientizados por la institución y en especial las autoridades, se verán reforzados si se hace una distribución equitativa y acorde a cada función de la carga académica de los docentes; debe ser tomado en cuenta su productividad y las actividades que realiza; es común escuchar decir que no se tienen suficientes horas por ejemplo para dedicarse a la investigación y esto tiende a dejar de lado proyectos o no querer participar en actividades que no están dentro de sus distributivos académicos. Si además de esto le sumamos una adecuada proporción de materiales y recursos y un espacio físico acorde a las características de la zona, se estará dando un paso hacia la mejora de la calidad, no solo de la educación sino también de todos los servicios brindados a la comunidad universitaria.

\subsection{Motivación laboral}

Para la dimensión motivación laboral (ML), relacionada con el estímulo interno del individuo, fueron valorados varios aspectos que se detallan a continuación (ver tabla 5).

\section{Tabla 5}

Preguntas procesadas por semaforización - dimensión ML

\begin{tabular}{|c|c|c|c|}
\hline Factor & Ítem (Toralmente de acuerdo) & Valoración & Lectura \\
\hline \multirow{6}{*}{ Compromiso } & $\begin{array}{l}\text { Dedicar más tiempo al trabajo del } \\
\text { que corresponde }\end{array}$ & $68,8 \%$ & Adecuado \\
\hline & $\begin{array}{l}\text { Sentir compromiso con la institución } \\
\text { por lo que ha recibido de ella }\end{array}$ & $84,4 \%$ & \multirow{5}{*}{ Óptimo } \\
\hline & Gusto por el trabajo que se realiza & $87,5 \%$ & \\
\hline & $\begin{array}{l}\text { Procura de no faltar al trabajo para } \\
\text { que la institución no se vea afectada }\end{array}$ & $87,5 \%$ & \\
\hline & $\begin{array}{l}\text { Considerar que se debe dar lo } \\
\text { mejor de sí a la institución por la } \\
\text { oportunidad de trabajar en ella }\end{array}$ & $87,5 \%$ & \\
\hline & $\begin{array}{l}\text { Aunque no sea pedido, gusto por } \\
\text { hacer bien el trabajo }\end{array}$ & $93,8 \%$ & \\
\hline
\end{tabular}




\section{Cont... Tabla 5}

\begin{tabular}{|c|c|c|c|}
\hline \multirow{7}{*}{ Identificación } & $\begin{array}{l}\text { De poder, traer traería familiares y } \\
\text { amigos a trabajar en la institución }\end{array}$ & $68,8 \%$ & \multirow{3}{*}{ Adecuado } \\
\hline & $\begin{array}{l}\text { Satisfacción porque otros sepan } \\
\text { que se trabaja en la institución }\end{array}$ & $75 \%$ & \\
\hline & $\begin{array}{l}\text { Sentirse cómodo y a gusto } \\
\text { trabajando en la institución }\end{array}$ & $75 \%$ & \\
\hline & $\begin{array}{l}\text { Si se escucha a desconocidos } \\
\text { hablar bien de la institución, el } \\
\text { sentimiento es de orgullo }\end{array}$ & $84,4 \%$ & \multirow{4}{*}{ Óptimo } \\
\hline & $\begin{array}{l}\text { Satisfacción por pensar que la } \\
\text { institución puede llegar a ser líder } \\
\text { en educación superior }\end{array}$ & $84,4 \%$ & \\
\hline & $\begin{array}{l}\text { Sentirse parte de la institución aún } \\
\text { fuera del horario de trabajo }\end{array}$ & $84,4 \%$ & \\
\hline & $\begin{array}{l}\text { Querer trabajar por largo tiempo en } \\
\text { la institución }\end{array}$ & $90,6 \%$ & \\
\hline \multirow{4}{*}{ Entusiasmo } & $\begin{array}{l}\text { Pensar pocas veces que el trabajo } \\
\text { es monótono y aburrido }\end{array}$ & $62,5 \%$ & \multirow{3}{*}{ Adecuado } \\
\hline & $\begin{array}{l}\text { Pertenecer a la institución hace que } \\
\text { se realice mejor esfuerzo }\end{array}$ & $71,9 \%$ & \\
\hline & Disfrute de la jornada de trabajo & $71,9 \%$ & \\
\hline & $\begin{array}{l}\text { Aunque a veces no se reciba } \\
\text { reconocimiento de los superiores, } \\
\text { se realiza el trabajo con } \\
\text { entusiasmo }\end{array}$ & $84,4 \%$ & Óptimo \\
\hline
\end{tabular}

Fuente: Elaboración propia.

Resalta el hecho de que los trabajadores, a pesar de los aspectos detectados relacionados al clima organizacional, reflejan estar altamente motivados, encontrándose en los resultados acciones a realizar a largo plazo o a mantener las existentes.

Uno de los estados adecuados está relacionado a la dedicación de mayor tiempo del que corresponde al trabajo, sin embargo, es saludable que los trabajadores den espacio a otros aspectos relacionados a otros ámbitos personales, y que de esta manera retornen a sus labores renovados para así tener un equilibrio en sus vidas entre el trabajo y la vida personal, sin caer en adicciones. Se sugiere, hacer capacitaciones de gestión eficaz del tiempo que permitan ser más eficientes y productivos, conseguir logros en un tiempo menor y tener menos estrés laboral, entre otros.

Otro estado adecuado es el relacionado con la identificación, cualidad que hace que los empleados se sientan comprometidos con su trabajo y que indique sentido de pertenencia para con la organización. Para Otzin, la identificación laboral en los trabajadores debe ser un compromiso, "el cual genera un mejor desempeño laboral en cada uno de ellos. Cuando un trabajador está identificado con la empresa, desarrolla sus funciones con interés logrando así un buen desempeño laboral" (2015: 1). 
Por otro lado, se tiene el entusiasmo detectado; este es una emoción que indica un estado de regocijo denotado por el interés en algo como el trabajo. Es de vital importancia mantener estos estados y reforzarlos a largo plazo. Se aconseja velar por las debidas pausas vacacionales, la buena comunicación, ajustarse a métodos de trabajo teniendo en cuenta el componente humano, reforzar lazos entre empleados y autoridades, promover la sana competitividad, edificar logros, adecuada capacitación, impulsar espacios comunes evitando aislamiento, entre otros.

Los demás factores encontrados están todos bajo la modalidad de mantenimiento, en estado óptimo, recomendándose hacerles seguimiento y mantener las acciones que hasta el momento han sido llevadas a cabo, reforzándolas.

Se aconseja, hacer estudios comparativos con evaluaciones de desempeño a través de co, auto y heteroevaluaciones, para así ver significativamente si el desempeño, valorado desde una perspectiva externa y desde el punto de vista de los resultados, refleja un sentir adecuado y óptimo. Estudiar si el clima organizacional detectado incide en los resultados de esta evaluación, pudiéndose agregar factores no tomados en cuenta en este estudio como el trabajo en equipo y descripción de puestos, además del desempeño laboral; aplicarlo en las demás unidades para un diagnóstico completo.

\section{Conclusiones}

El clima organizacional es el conjunto de percepciones que el colectivo tiene sobre el medio físico y humano donde se desempeñan; revelan los anales de lo que sucede a lo interno, sus procesos, el tipo de personas, su comunicación, infraestructura y como es ejercida la autoridad; aspectos estos externos al trabajador.

La motivación laboral es lo que impulsa por voluntad propia al trabajador a querer alcanzar objetivos y metas; impulsos enfocados en satisfacer sus necesidades mediante el mantenimiento de conductas durante el ejercicio de labores; aspectos estos internos del trabajador.

La técnica de semaforización es útil para el diagnóstico y medición de factores que inciden en el clima organizacional y motivación laboral; permite valorar estos parámetros y determinar el tiempo en que deben darse los cambios y su profundidad.

El porcentaje de participación de las mujeres es menor en las ingenierías, tanto a nivel de estudio como docente, por lo que debe impulsarse cambios en estos paradigmas y romper las brechas de género.

El clima organizacional se ve afectado por las condiciones de los puestos de trabajo, ya que del confort con que se desempeñen las actividades dependerá el éxito de ellas. Fomentar el compañerismo y competitividad sana entre trabajadores hacen que el clima organizacional mejore; además de la implementación de modelos de compensación laboral que promuevan la retención de personal calificado y una mejora en la calidad del servicio, valiéndose de un alza en remuneraciones y en retribuciones indirectas.

La evaluación institucional debe enfocarse en mejoras profundas a nivel académico y personal y mitigar debilidades, desprendiéndose de ella planes de capacitación continuos, basados en necesidades reales, 
orientados al logro de objetivos organizacionales. Se deben establecer liderazgos participativos y transformacionales. Ver a las autoridades como pares, conllevará a una mejora de relaciones y fomentar el sentido de inclusión.

La capacitación en gestión eficaz del tiempo hará que los trabajadores logren un equilibrio entre el trabajo y vidas personales, hacer un uso eficiente y eficaz del tiempo, repercutiendo así en mayor productividad.

La identificación y el sentido de pertenencia generan un compromiso en el trabajador; el mantener el entusiasmo le permite la mejora del espacio compartido, favorece el clima organizacional y facilita el trabajo colaborativo.

No siempre el clima organizacional incide en la motivación laboral, donde a pesar de los problemas detectados en el caso de estudio, los trabajadores reflejan alta motivación.

Los diagnósticos realizados a las organizaciones deben servir de insumos para la elaboración de planes de mejora que se centren en los aspectos desfavorables encontrados. Las acciones que provengan de factores en estado crítico deberán estar basadas en un plan de contingencia de implementación inmediata; aquellos en estado de riesgo, preventivo y adecuado, requieran acciones a corto, largo y mediano plazo respectivamente. No deben descuidarse los factores encontrados en estado óptimo, debiendo mantenerse y ser reforzados.

\section{Referencias Bibliográficas}

Alfaro, J. (2019). Clima organizacional y satisfacción laboral en el Ministerio Público en el Distrito Fiscal de
Ventanilla (Tesis de grado). Universidad César Vallejo, Perú.

Albarracin, P., y Gómez, M. (2021). Evaluación anual de desempeño docente como fundamento para la mejora de la práctica pedagógica de los profesores del Colegio Almirante Padilla IED. https://repository.ucc. edu.co/handle/20.500.12494/33219

Álvarez, B. \& Ayala, J. (2020). Factores que influyen en la motivación laboral de trabajadores en empresas de telecomunicaciones. Una revisión teórica (Tesis de grado inédita). Universidad Peruana Unión, Perú.

Angulo, P. (2017). Encuesta 2017 a graduados de la Escuela Politécnica Nacional, carrera Ingeniería Civil. Ecuador: Escuela Politécnica Nacional.

Arreiza, J., Restrepo, S. \& Osorio, U. (2020). Motivación y tecnología, aspectos claves para mejorar el desempeño laboral del docente. En J. Sepúlveda (ed.). Emociones y liderazgo en el desempeño laboral y el emprendimiento, (pp. 87-104). Colombia: Sello Editorial Universitario Americana.

Atoche, M. (2019). Relaciones interpersonales y motivación laboral de los docentes de la I.E Fe y Alegría $N^{\circ}$ 18, Sullana - Piura. (Tesis de grado). Universidad César Vallejo, Perú.

Berlanga, K., Balderas, A., Barrios, S. \& Cruz, C. (2020). Análisis del avance en formación docente en el TECNM Campus Ciudad Valles para una formación ideal. Tectzapic, 6(2), 96104.

Borja, J. (2021). Plan de comunicación interna en Pisende S.A.S. (Tesis de grado). Corporación Universitaria Lasallista, Colombia. 
Castiblanco, L., Lozano, L., Mora, M. \& Camargo, S. (2020). Propuesta plan de mejora del clima organizacional en la empresa SIQUIMA EXPRESS S.A., para el cuarto trimestre 2020. (Ensayo de diplomado). Universidad Nacional Abierta y a Distancia, Colombia.

Castillo, C. (2021). Motivación y desempeño laboral de los docentes de las Direcciones Distritales de Educación. (Tesis de maestría). Universidad Técnica de Ambato, Ecuador.

Castillo, J. (2018). Motivación laboral y Productividad de la fuerza de ventas de TRIATHLON SAC. (Tesis de grado). Universidad César Vallejo, Perú.

Cetzal, R., Mac, C., Ramírez, C. \& Osuna, N. (2020). Factores que Afectan el Desempeño Docente en Centros de Alta y Baja Eficacia en México. REICE, 18(2), 77-95. https:// doi.org/10.15366/reice2020.18.2.004

Cevallos, L. \& Rodríguez, A. (2018). Estrategia Para Mejorar el Desempeño Laboral de las Secretarias de Facultad en las Unidades Académicas de Universidad Laica Eloy Alfaro de Manabí, de la Ciudad de Manta. Ecuador: Consejo Editorial.

Chávez, R., Escudero, J., \& Delfín, L. (2016). El origen del clima organizacional, desde una perspectiva de las escuelas de la administración: una aproximación. México: Universidad Veracruzana.

Chelton, K. \& Balladares, E. (2020). Satisfacción del trabajador y su incidencia en el desempeño laboral, Municipalidad Distrital de Pampas de Hospital, Tumbes, 2019. (Tesis de grado). Universidad Nacional de Tumbes, Perú.
Chimbosina, V. (2021). Marketing de influencers como estrategia de posicionamiento de marca en la empresa de sandalias tradicionales Tayta Berna (Tesis de grado). Universidad Técnica de Ambato, Ecuador.

Delgado, M. (2020). Identidad Corporativa y Motivación Laboral en Enfermeras de un Hospital en el Callao (Tesis de grado). Universidad Peruana de Ciencias Aplicadas, Perú.

Espinoza, J. (2020). Motivación a los empleados del Comercial "La Caraguay Chiquita de Ricaute" (Tesis de grado inédita). Universidad Técnica de Babahoyo, Ecuador.

Fassio, A. \& Pascual, L. (2016). Apuntes para desarrollar una investigación en el campo de la administración y el análisis organizacional. Argentina: Editorial Universitaria de Buenos Aires.

Feixas, M. (2006). Cuestionario para el análisis de la orientación docente del profesor universitario. Revista de Investigación Educativa, 24(1), 97118.

Flores, C., Fernández, M., Juárez, A., Merino, C., \& Guimet, M. (2015). Entusiasmo por el trabajo (engagement): un estudio de validez en profesionales de la docencia en Lima, Perú. Liberabit, 21(2), 195-206.

García, B. (2019). Liderazgo transformacional y calidad educativa en el CEBA Augusto B. Leguía en el distrito de Puente Piedra 2019. (Tesis de maestría). Universidad César Vallejo, Perú

Gedesa. (2019). Productividad laboral, ¿cómo influye el mobiliario de oficina? https://bit.ly/3dxnk55

Hernández, R., Fernández, C. y 
Baptista, M. (2010). Metodología de la investigación. McGrawHill. https:// bit.ly/3n12oGN

Instituto Nacional de Meteorología e Hidrología. (2020). Boletín climatológico mensual - diciembre 2020. Quito, Ecuador: INAMHI.

Jiménez, H. y Mosquera, A. (2017). Clima organizacional y su incidencia en el desempeño laboral de los trabajadores, de los departamentos financieros en entidades públicas. (Tesis de grado inédita). Universidad del Pacífico, Quito, Ecuador.

Lainez, J. (2019). Influencia del clima organizacional en el desempeño docente de una Unidad Educativa, Santa Elena, Ecuador. (Tesis de grado). Universidad César Vallejo, Perú.

Maldonado, T. (2020). Incidencia de la carga de trabajo en la satisfacción laboral del personal de la Dirección de Talento Humano de la Pontificia Universidad Católica del Ecuador (PUCE) en el año 2019. (Tesis de maestría). Universidad Andina Simón Bolívar, Sede ecuador.

Malhotra, N. (2004). Investigación de Mercados. México: Pearson Educación.

Manzano, E. (2020). La educación empresarial en gestión de operaciones integrada al desarrollo de emprendimientos innovadores. (Trabajo de titulación modalidad artículo). Universidad Técnica de Ambato, Ecuador.

Martínez, C. (2014). Técnicas e instrumentos de recogida $y$ análisis de datos. España: Universidad Nacional de Educación a Distancia.

Mina, C. (30 de marzo de 2018). Temperatura más alta en Portoviejo. El Diario, s.p.
Múria, J., y Gil, R. (1998). Preparación, tabulación y análisis de encuestas para directivos. Madrid: ESIC.

Moreno, V. (2019). Gestión de Recursos Humanos. España: IC editorial.

Núñez, J. (2007). Formación docente y saberes locales: una mirada desde la ruralidad. Venezuela. Revista Electrónica Educare, 2, 25-32. https://doi.org/10.15359/ree.2-Ext.1

Otzin, C. (2015). Factores de identificación y orgullo laboral en trabajadores del Departamento de Seguridad Integral en Empresa Portuaria Quetzal (Tesis de grado). Universidad Rafael Landívar, Guatemala.

Palma, M. \& Hermosillo, L. (2020). Satisfacción laboral y del cliente de una empresa comercial. En G. Córdova (coord.). La práctica y la investigación universitaria aplicada, (pp. 55-69). México: Instituto Tecnológico de Sonora.

Pecino-Medina, V., Mañas-Rodríguez, M., Díaz-Fúñez, P., López-Puga, J., \& Llopis-Marín, J. (2015). Clima y satisfacción laboral en el contexto universitario. Anales de psicología, 31(2), 658-666. $\quad$ http://dx.doi. org/10.6018/analesps.31.2.171721

Peña, C. (2015). La motivación laboral como herramienta de gestión en las organizaciones empresariales (Tesis de grado). Universidad Pontificia Comillas ICAI- ICADE, España.

Peña, H. \& Villón, S. (2018). Motivación Laboral. Elemento Fundamental en el Éxito Organizacional. Revista Scientific, 3(7), 177-192.

Pérez, F. (2014). El Clima Organizacional y su incidencia en el desempeño laboral de los trabajadores del MIES - Dirección Provincial Pichincha (Tesis de grado). Universidad Central 
del Ecuador, Quito, Ecuador.

Pichón, C. \& Vega, M. (2020). El trabajo en casa, medida de emergencia ante la situación de covid-19 en Barranquilla que vulnera las condiciones de trabajo del empleado (Tesis de maestría). Universidad Simón Bolívar, Colombia.

Ramírez, R.; Manosalvas, M. \& Cárdenas, O. (2019). Estereotipos de género y su impacto en la educación de la mujer en Latinoamérica y el Ecuador. Espacios, 40(41), 29-36.

Ramos, D. (2012). El Clima Organizacional, definición, teoría, dimensiones y modelos de Abordaje. (Tesis de grado). Universidad Nacional Abierta y a Distancia, Colombia.

Reguant, M. y Martínez-Olmo, F. (2014). Operacionalización de conceptos/ variables. Barcelona, España: Depósito Digital de la UB.

Riquelme, M. (2021). ¿Cómo influye el clima en la productividad laboral? https://bit.ly/3v2DTfb

Rojas, F. (2018). Capacitación y desempeño laboral (Tesis de grado). Universidad Rafael Landívar, Guatemala.

Rosales, B. (2020). Análisis anual de los principales indicadores de educación superior, ciencia, tecnología e innovación. https://bit.ly/3dwccW6

Ruiz, C. (2020). Incidencia de la turnicidad y exposición a ruido en la fatiga laboral en una empresa de gestión de residuos de los Ángeles, Chile. (Tesis de grado). Universidad de Concepción, Chile.

Solórzano, K. (2020). Análisis de incentivos como herramienta motivacional para el personal de las Unidades Administrativas de la Dirección Provincial de Esmeraldas del Consejo de la Judicatura. (Tesis doctoral inédita). Ponticifia Universidad Católica Sede Esmeraldas, Ecuador.

Tapia, L. (2020). El reconocimiento laboral y su relación con la rotación del personal en la generación Millennial de las empresas del sector norte de Quito. (Tesis de maestría inédita). Universidad Técnica de Ambato, Ecuador.

Torres, E. \& Espino, A. (2020). Percepción del clima organizacional de los trabajadores docentes en el Perú: una revisión teórica. (Trabajo de grado). Universidad Peruana Unión, Perú.

Valdez, N. (2013). Proceso de asignación de recursos a las IES y su impacto en la calidad educativa de la Universidad Pública Sinaloense, un estudio de caso. (Tesis doctoral). Universidad Pública Sinaloense, México.

Villalba, M. (2020). Empoderamiento en las autoridades de la Unidad Educativa León Febres Cordero. Universidad Técnica de Babahoyo, Ecuador.

Weather Spark. (2021). El clima en Portoviejo. https://bit.ly/3dxWRV8

Yamin, J., Pattini, A. \& Colombo, E. (2020). Confort visual en oficinas, factor temporal en la evaluación de deslumbramiento. Informes de la construcción, 72, 1-8.

Zegarra, F. (2021). Estilos de Liderezgo y Clima Organizacional en los Profesores de los Centros Educativos del Sistema FAP Región Sur - Arequipa 2019. https://bit. ly/3x7RKTp 